\title{
Síntesis in situ de nanopartículas de peróxido de zinc sobre fibras de plátano con potencial en la industria textil
}

\section{In situ synthesis of nanoparticles of zinc peroxide on banana fibers with potential in the textile industry}

María Estefanía Ballesteros García Departamento de Ingeniería, Facultad de Ciencias Naturales e Ingeniería, Universidad de Bogotá Jorge Tadeo Lozano, Bogotá, D. C., Colombia John Ricardo Jr. Durán Romero Departamento de Ingeniería, Facultad de Ciencias Naturales e Ingeniería, Universidad de Bogotá Jorge Tadeo Lozano, Bogotá, D. C., Colombia Juliana Gutiérrez Corrales

Programa de Diseño y Gestión de la Moda, Facultad de Artes y Diseño, Universidad de Bogotá Jorge Tadeo Lozano, Bogotá, D. C., Colombia Alis Pataquiva Mateus Departamento de Ingeniería, Facultad de Ciencias Naturales e Ingeniería, Universidad de Bogotá Jorge Tadeo Lozano, Bogotá, D. C., Colombia http://orcid.org/0000-0003-2953-5132 alisy.pataquivam@utadeo.edu.co

Fecha de recepción: 1 de septiembre de 2017 Fecha de aceptación: 5 de octubre de 2017

Sugerencia de citación: Ballesteros García, M. E., Durán Romero, Jr., Gutiérrez Corrales, J. y Pataquiva Mateus, A. (2017). Síntesis in situ de nanopartículas de peróxido de zinc sobre fibras de plátano con potencial en la industria textil. Mutis 7(2), 23-32, doi: http://dx.doi.org/10.21789/22561498.1247

Editor: Hernández Fernández, J. javier.hernandez@utadeo.edu.co

\section{RESUMEN}

El presente trabajo de investigación plantea un desarrollo en torno al manejo de residuos en los cultivos de plátano, siendo una idea sostenible con respecto a la extracción y adquisición de fibras con cualidades innovadoras que posean nanopartículas (NPs) de $\mathrm{ZnO}_{2}$ como la capacidad antimicrobiana. La finalidad de la investigación fue la de nanoestructurar fibras provenientes de residuos del plátano con NPs de peróxido de zinc como una propuesta sostenible, teniendo en cuenta factores de optimización en procesos para la síntesis de las nanopartículas. Los resultados más relevantes se obtuvieron en la prueba física microscopia electrónica de barrido (SEM), en donde se destaca el tamaño nanométrico de las partículas sintetizadas de $94 \mathrm{~nm}$. Se demostró el factor hidrofóbico de las fibras de plátano por encima de las fibras de algodón.

Palabras clave: Nanopartículas, Plátano, Fibras, Residuos agroindustriales, dióxido de zinc 


\section{ABSTRACT}

This research proposes a development around the management of residues in banana crops, being a sustainable idea regarding the extraction and acquisition of fibers with innovative qualities that possess $\mathrm{ZnO} 2$ nanoparticles (NPs) as the antimicrobial capacity. The purpose of this research was to nanostructure fibers from banana waste with zinc peroxide NPs as a sustainable proposal, taking into account optimization factors in processes for the synthesis of nanoparticles. The most relevant results were obtained in the physical scanning electron microscopy (SEM), where the nanometer size of the synthesized particles was $94 \mathrm{~nm}$. The hydrophobic factor of the banana fibers was shown above cotton fibers.

Keywords: Nanoparticles, Banana, Fibers, Agroindustrial wastes, zinc dioxide

\section{INTRODUCCIÓN}

En Colombia, anualmente se producen 3'379.742 toneladas de plátano, de las cuales se generan 53.250 toneladas de residuos provenientes de la práctica agronómica de corte y mantenimiento de la musa de plátano. La gran mayoría de residuos agroindustriales ocasionan un alto impacto ambiental, ya que la mayoría de estos son incinerados y usados como cenizas aportando material de abono para suelos cafeteros (DANE, 2014).

En busca de nuevas materias primas para la producción de textiles se ha encontrado que a partir de los residuos de plátano se pueden obtener fibras con un alto porcentaje de celulosa y resistencia mecánica (Alarcón, López y Restrepo, 2013). Las fibras de plátano son adecuadas para la implementación de nanopartículas de $\mathrm{ZnO}_{2}$, ya que poseen una superficie porosa innata que permiten la conexión entre nanopartículas y fibra, además de esto su estructura nanoporosa contempla una alta densidad de oxígeno que funciona como un reactor que forma y estabiliza las nanopartículas de manera equilibrada durante todo el tejido (Alarcón, López, y Restrepo, 2013)

Hoy en día, la nanoestructuración de fibras a partir de residuos de plátano no se ha desarrollado en ningún campo, debido a la poca investigación sobre la fibra sin dudar de que sus propiedades naturales se rescatan resultados asombrosos de resistencia y capacidad hidrofóbica (Colonia, Martínez, y Solís, 2013).
El objetivo del presente trabajo fue la estructuración de fibras provenientes del beneficio del plátano con nanopartículas de peróxido de zinc (NPs de $\mathrm{ZnO}_{2}$ ) como una propuesta sostenible en el sector textil.

\section{MATERIALES Y MÉTODOS}

A continuación, se presentarán detalladamente los métodos y materiales que se usaron a lo largo de la experimentación.

\section{Pretratamiento de la fibra de plátano}

Las fibras de plátano fueron obtenidas a partir de un proceso de desfibración, en donde el seudotallo de la musa de plátano fue sometido a un proceso de compresión a través de rodillos. Este proceso retiró la humedad y lentamente se fueron obteniendo las fibras, trabajo que actualmente es llevado a cabo por madres cabeza de familia en el departamento de Caldas, Colombia. Al iniciar el proceso, las fibras se limpiaron de tal forma que se eliminaron residuos vegetales o algún otro tipo de impurezas. Después se sometieron a un baño con agua destilada a $70{ }^{\circ} \mathrm{C}$ durante $1 \mathrm{~h}$. Posteriormente, fueron secadas en un horno de temperatura constante de $80^{\circ} \mathrm{C}$ durante 45 min (Ovalle, Blanco-Tirado, y Combariza, 2013). 


\section{Síntesis de nanopartículas de $\mathrm{ZnO}_{2}$}

La síntesis de las NPs de $\mathrm{ZnO}_{2}$ fue basado en el trabajo de Colonia y sus colaboradores (Colonia et al., 2013). Brevemente, se trabajó por medio de la ruta sol-gel, método por el cual el tejido a base de residuos de plátano adquirió de manera más homogénea las NPs de $\mathrm{ZnO}_{2}$, para ello se preparó una solución precursora donde inicialmente se usaron $5 \mathrm{~mL}$ de $\mathrm{H}_{2} \mathrm{O}_{2}$ al $30 \%$ con $50 \mathrm{~mL}$ de $\mathrm{H}_{2} \mathrm{O}$ y se sonicó la solución por 2 min. Posteriormente, se mezcló $1 \mathrm{~g}$ de acetato de zinc di-hidratado en la solución de $\mathrm{H}_{2} \mathrm{O}_{2}$ por 5 min con el objetivo de lograr una solución homogénea. La fibra fue sumergida y sonicada entre 30 y 90 min con el objetivo de instituir las NPs $\mathrm{ZnO}_{2}$. Seguidamente, se procedió a centrifugar a $4000 \mathrm{rpm}$ durante $30 \mathrm{~min}$ obteniendo separar las partículas de gel formadas de coloide preliminar síntesis descrita en el procedimiento se rige por la siguiente reacción redox:

$$
\begin{gathered}
\left(\mathrm{Zn}\left(\mathrm{CH}_{3} \mathrm{COO}\right) 2.2 \mathrm{H}_{2} \mathrm{O}(\mathrm{ac})+\mathrm{H}_{2} \mathrm{O}_{2}\right. \text { Ultrasonido } \\
\left.\mathrm{ZnO} \mathrm{O}_{2} \downarrow+2\left(\mathrm{CH}_{3} \mathrm{COOH}\right)(\mathrm{ac})+2 \mathrm{H}_{2} \mathrm{O}\right)
\end{gathered}
$$

Finalmente, las partículas formadas se lavaron dos veces con agua destilada y se secaron a $80^{\circ} \mathrm{C}$ por $12 \mathrm{~h}$.

\begin{tabular}{|c|c|c|c|c|c|c|c|}
\hline Factor & r Nombre & nidades & Tipo & Mínim & Máxim & Media & $\begin{array}{l}\text { Desviación } \\
\text { estándar }\end{array}$ \\
\hline A & {$\left[\mathrm{H}_{2} \mathrm{O}_{2}\right]$} & $\%$ & Numérico & 30 & 50 & 40 & 7,07 \\
\hline$B$ & T adición & Min & Numérico & 30 & 130 & 80 & 35,36 \\
\hline C & T Sonic. & $\min$ & Numérico & 4 & 6 & 4,94 & 0,66 \\
\hline
\end{tabular}

Tabla 1. Factores influyentes en el diseño experimental

\section{Diseño experimental y análisis estadístico}

El diseño experimental a evaluar fue el Box-Behnken (Design Expert 6.0.8), ya que este permitió evaluar una superficie de respuesta a partir de la evaluación de diferentes mezclas de condiciones fundamentales en la síntesis de las nanopartículas. El software permitió modelar la metodología a lo largo del proyecto para obtener resultados óptimos y mejorados sobre los procesos planteados inicialmente. El modelo de diseño fue de tipo cuadrático en donde este se ajusta a un experimento secuencial y otorga la creación de factores según su importancia en la optimización del proceso. Las variables escogidas para realizar el diseño experimental fueron: A: Concentración de peróxido (\%), B: Tiempo posterior a la adición del acetato de zinc di-hidratado ( $\mathrm{min}), \mathrm{C}$ : Tiempo de sonicación (min); con respuesta de diámetro de partícula. Los valores del rango estudiado para cada variable se encuentran en la Tabla 1 y en la Tabla 2 se describen las 17 corridas, que se llevaron a acabo aleatoria, y que arroja el diseño experimental para estas tres variables.

Tabla 2. Diseño Experimental con factores variables. A: Concentración de peróxido (\%), B: Tiempo posterior a la adición del acetato de zinc dihidratado, C: Tiempo de sonicación

\begin{tabular}{|c|c|c|c|}
\hline Run & $\begin{array}{c}\text { Factor 1 } \\
\text { A: Ca } \\
\%\end{array}$ & $\begin{array}{c}\text { Factor 2 } \\
\text { B: TimeS1 } \\
\text { min }\end{array}$ & $\begin{array}{c}\text { Factor 3 } \\
\text { C: TimeS2 } \\
\text { min }\end{array}$ \\
\hline 1 & 40 & 130 & 4 \\
\hline 2 & 30 & 80 & 5 \\
\hline 3 & 30 & 80 & 4 \\
\hline 4 & 40 & 80 & 5 \\
\hline 5 & 50 & 130 & 5 \\
\hline 6 & 40 & 80 & 5 \\
\hline 7 & 40 & 30 & 6 \\
\hline 8 & 40 & 30 & 4 \\
\hline 9 & 40 & 80 & 5 \\
\hline 10 & 50 & 80 & 4 \\
\hline 11 & 40 & 80 & 5 \\
\hline 12 & 50 & 80 & 6 \\
\hline 13 & 40 & 80 & 5 \\
\hline 14 & 30 & 130 & 5 \\
\hline 15 & 50 & 30 & 5 \\
\hline 16 & 40 & 130 & 6 \\
\hline 17 & 30 & 30 & 5 \\
\hline
\end{tabular}




\section{CARACTERIZACIÓN DE FIBRAS NANOESTRUCTURADAS CON ZNO}

\section{Microscopía electrónica de barrido}

La morfología del material y la determinación de los elementos presentes en la superficie se realizaron por microscopía electrónica de barrido (SEM) y espectroscopía de rayos $X$ por energía dispersiva (EDS) respectivamente. Las fibras deben ser recubiertas con de oro para luego visualizar las nanopartículas con el equipo SEM Hitachi TM-1000, en una aceleración de electrones de $5 \mathrm{kV}$ y diferentes magnificaciones que oscilarán entre 100 kX y 200 kX.

\section{Hidrofobicidad textil}

Este método permitió conocer la tensión superficial que tiene la fibra de plátano frente al agua, este consistió en dejar caer una gota de agua y después de 60 s se tomó una fotografía y posteriormente, con ayuda de ImageJ (Schneider, Rasband, y Eliceiri, 2012) se determinó su ángulo de contacto.

\section{Resistencia de NPs de $\mathrm{ZnO}_{2}$ a la fibra después del lavado}

Esta técnica empírica consistió en hacer un lavado prolongado durante $1 \mathrm{~h}$, esto se desarrolló empleando una lavadora Samsung $16 \mathrm{~L}$, las fibras nanoestructuradas se sometieron a un ciclo de lavado normal con una concentración inicial de detergente en polvo comercial para ropa al $40 \%$. Posteriormente, se llevó al microscopio electrónico de barrido (SEM) y así se logró visualizar la permanencia de NPs de $\mathrm{ZnO} 2$ en las fibras.

\section{Sensibilidad microbiana sobre fibras textiles}

Este método permitió comprobar las propiedades antimicrobianas del textil y se realizó mediante la com- paración del crecimiento de microorganismos entre el tejido original (tejido de control) y el tejido tratado con las NPs de $\mathrm{ZnO}_{2}$. Su objetivo fue determinar la actividad antimicrobiana de los materiales textiles a ser evaluados. Se utilizó un método de difusión en agar y como inóculo caldos de Escherichia coli. El agar se dispuso sobre cajas Petri estériles y se sembraron los microorganismos sobre la superficie de las placas de agar. Las fibras tratadas con las NPs $\mathrm{ZnO} 2$ y las fibras de control se presionaron en el centro del cultivo, para adherir los microorganismos al tejido (Rajendra et al., 2010).

Finalmente, las placas se incubaron a $37^{\circ} \mathrm{C}$ durante 48 horas. La formación de una zona claramente delimitada interrumpió el crecimiento microbiano, esto se creó alrededor de la muestra que indicó la actividad antibacteriana de la fibra tal cual como lo había documentado Herrera (Herrera, 1999) en sus pruebas de sensibilidad antimicrobiana en dónde en donde describió que el halo mínimo de resistencia es de 2 a $3 \mathrm{~mm}$.

\section{Propiedades de tracción de fibras textiles simples}

Esta prueba siguió la norma ASTM D3822-07, empleando la máquina universal (Jinan Testing Equipment IE Corporation), en donde se obtuvieron los valores de resistencia y tensión $(n=5)$.

\section{RESULTADOS Y DISCUSIÓN}

Los resultados de la En respuesta de optimización y manteniendo las características principales del proyecto, se obtuvo por medio de la microscopía electrónica de barrido (SEM) que las muestras que se encuentran en un rango de 0 a $100 \mathrm{~nm}$ fueron las muestras 7 y 16 con diámetro de partícula de $94.10 \mathrm{~nm}$ y $94.12 \mathrm{~nm}$, respectivamente. 
Tabla 3. Diseño Experimental Resultados obtenidos de (SEM) después del análisis estadístico de cada una de las muestras nanoestructuradas

\begin{tabular}{|c|c|c|c|c|c|}
\hline Std & Run & $\begin{array}{c}\text { Factor 1 } \\
\text { A: Ca } \\
\%\end{array}$ & $\begin{array}{c}\text { Factor 2 } \\
\text { B: TimeS1 } \\
\text { min }\end{array}$ & $\begin{array}{c}\text { Factor 3 } \\
\text { C: TimeS2 } \\
\text { min }\end{array}$ & $\begin{array}{c}\text { Response 1 } \\
\text { Tamaño NPs } \\
\text { ZnO2 } \\
\text { nm } \\
\text { (n=50) }\end{array}$ \\
\hline 10 & 1 & 40 & 130 & 4 & 293,408 \\
\hline 7 & 2 & 30 & 80 & 5 & 145,584 \\
\hline 5 & 3 & 30 & 80 & 4 & 103,689 \\
\hline 15 & 4 & 40 & 80 & 5 & 105,561 \\
\hline 4 & 5 & 50 & 130 & 5 & 115,537 \\
\hline 13 & 6 & 40 & 80 & 5 & 123,118 \\
\hline 11 & 7 & 40 & 30 & 6 & 94,0966 \\
\hline 9 & 8 & 40 & 30 & 4 & 163,698 \\
\hline 14 & 9 & 40 & 80 & 5 & 139,199 \\
\hline 6 & 10 & 50 & 80 & 4 & 188,938 \\
\hline 17 & 11 & 40 & 80 & 5 & 155,935 \\
\hline 8 & 12 & 50 & 80 & 6 & 193,437 \\
\hline 16 & 13 & 40 & 80 & 5 & 132,762 \\
\hline 3 & 14 & 30 & 130 & 5 & 162,243 \\
\hline 2 & 15 & 50 & 30 & 5 & 175,689 \\
\hline 12 & 16 & 40 & 130 & 6 & 94,0799 \\
\hline 1 & 17 & 30 & 30 & 5 & 98,1198 \\
\hline
\end{tabular}

Figura 1. Superficie de respuesta para el error estándar de la experimentación

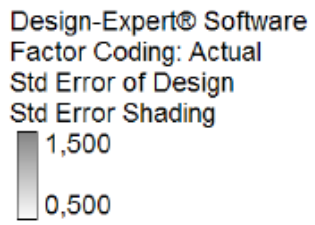

Std Error of Design $=0,901$ Std \# 12 Run \# 16 $\mathrm{X} 1=\mathrm{A}: \mathrm{Ca}=40,00$ X2 = B: TimeS1 $=130,00$

Actual Factor

C: TimeS2 $=6,00$

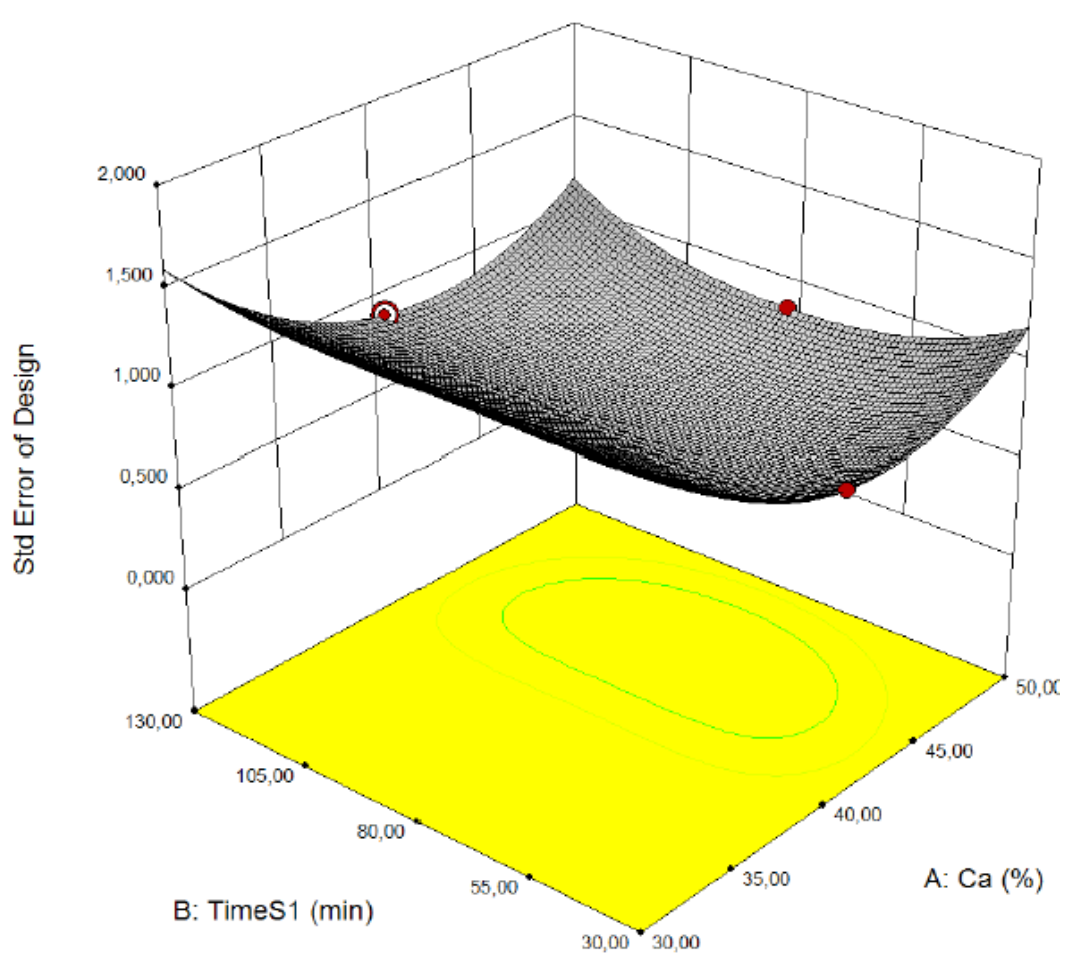


Según los resultados obtenidos del diseño experimental tal cual como se muestra en la Tabla 3, se pueden observar las variaciones en la metodología de Colonia y colaboradores (Colonia et al., 2013), en donde se modificaron las cantidades de reactivos e intervalos de tiempo que se usaron en la síntesis de las nanopartículas de peróxido de zinc. Las mayores afectaciones del tamaño de las nanopartículas, se dieron en cuanto al factor 3 que es el tiempo (min) de sonicación posterior a la mezcla acetato de zinc di-hidratado en la solución de $\mathrm{H}_{2} \mathrm{O}_{2}$, resultando ser un factor que garantiza la homogeneidad de la mezcla antes de la sonicación final.

Por otro lado, se determinó que la concentración óptima de $\mathrm{H}_{2} \mathrm{O}_{2}$ al iniciar el proceso está en un rango del 30 al $40 \%$ ya que, los resultados del tamaño de las nanopartículas estuvieron por debajo de los $100 \mathrm{~nm}$. Para la superficie de respuesta que presenta la experimentación, se tiene la Figura 1 en donde se obtuvo una región óptima de concentración de $\mathrm{H}_{2} \mathrm{O}_{2}$ que se encontró entre 33 - 42\% y el tiempo de sonicación final de 35 - 91 min. Para el diseño experimental se obtuvo una zona de probabilidad ascendente en cuanto a estudios evaluados según el tamaño de nanopartículas que relaciona una probabilidad de éxito mayor al $90 \%$ en la presente metodología.

Figura 2. Fibras de plátano sin nanoestructurar

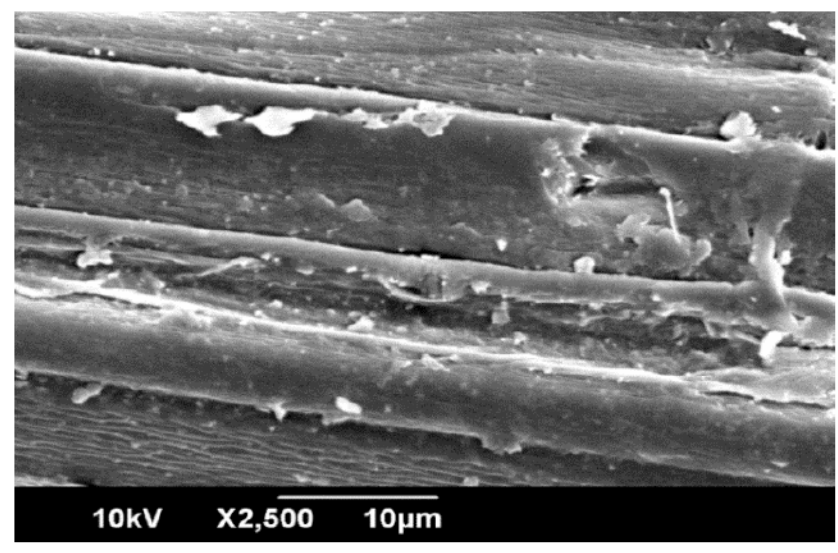

Figura 3. Fibra de plátano con NPs de $\mathrm{ZnO}_{2}$ (Muestra16)

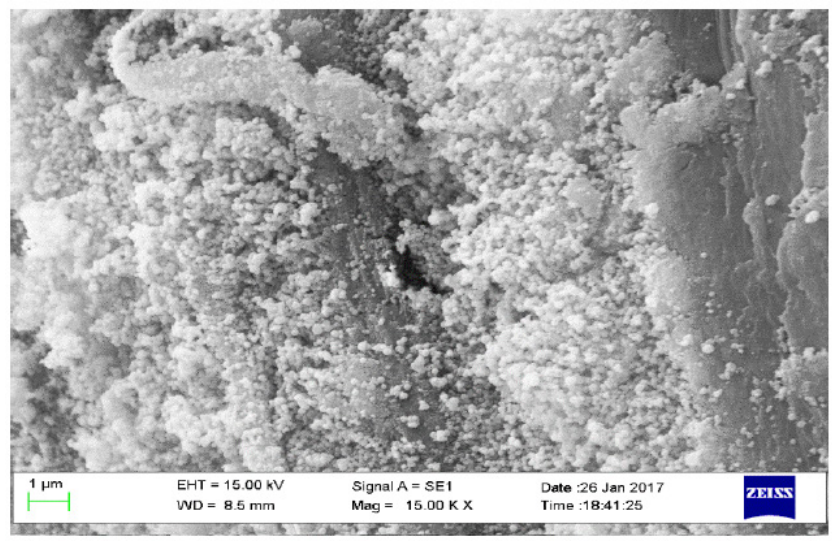

Se visualizó la fibra de plátano sin nanoestructurar Figura 2 para lograr ver su estructura superficial, en donde se caracterizó por contener un tipo carrileras que por su tamaño poseen una profundidad que facilita la adherencia de las nanopartículas. A partir de los resultados obtenidos para la visualización de NPs de $\mathrm{ZnO}_{2}$ sobre la fibra (Figura 3), se observó que las nanopartículas recubren en buena medida la superficie de la fibra en forma de agrupaciones de nanopartículas (clusters).

Figura 4. Tensión superficial de la fibra de plátano

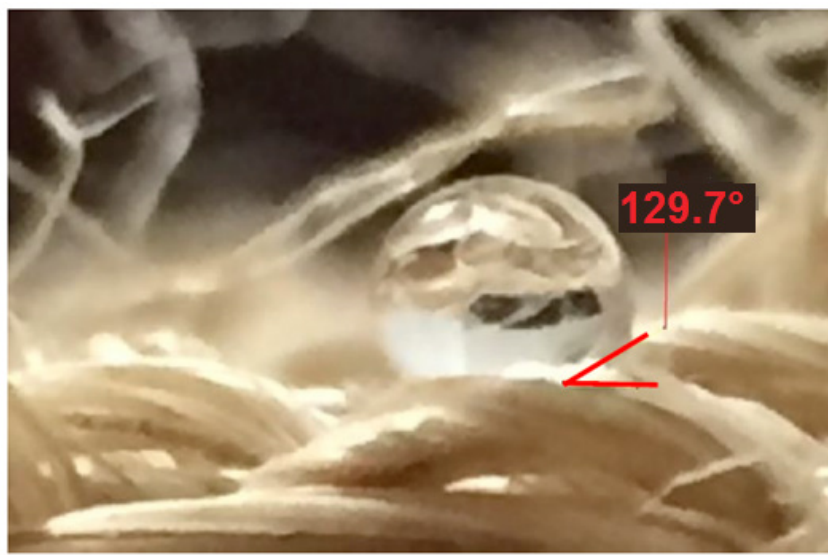


Figura 5. Tensión superficial de la fibra de algodón

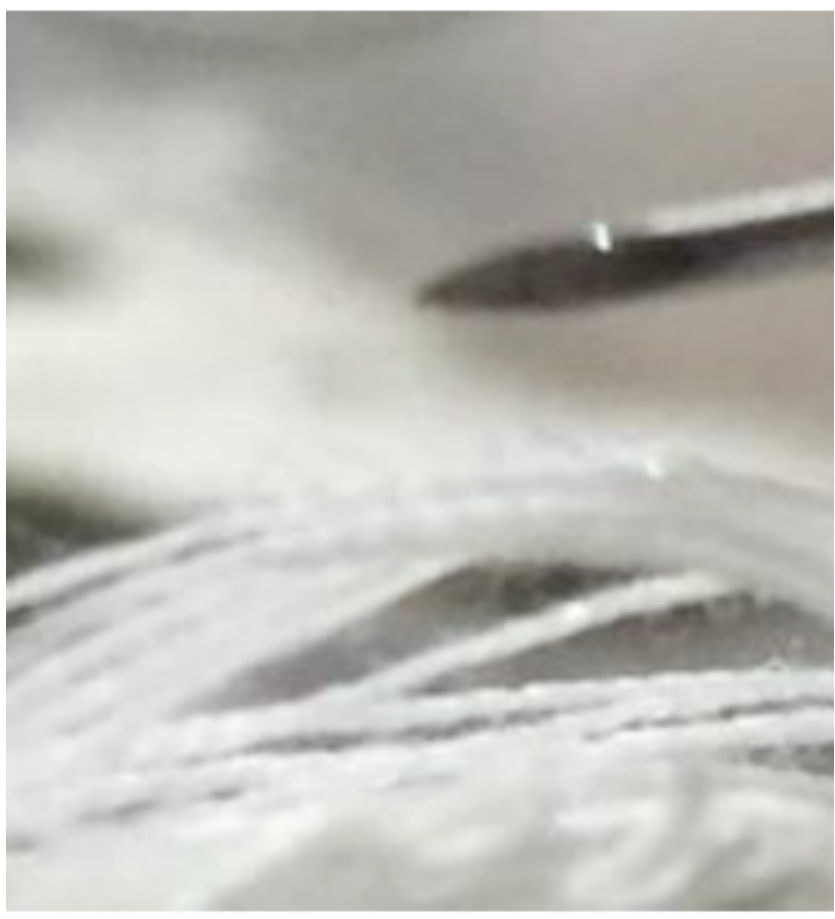

Tabla 4. Resultados obtenidos después del análisis estadístico de la fibra de plátano (Fuerzas intermoleculares)

\begin{tabular}{lllll}
\hline I & $\theta^{\circ}$ Aire & $\begin{array}{l}\text { Grado de } \\
\text { mojabilidad }\end{array}$ & $\begin{array}{l}\text { Interacciones } \\
\text { S/L }\end{array}$ & $\begin{array}{l}\text { Interacciones } \\
\mathrm{L} / \mathrm{L}\end{array}$ \\
\hline 1 & $44,878+/-0,3^{\circ}$ & Baja & Débil & Fuerte \\
2 & $39,703+/-0,2^{\circ}$ & Baja & Débil & Fuerte \\
3 & $32,209+/-0,4^{\circ}$ & Baja & Débil & Fuerte \\
\hline
\end{tabular}

Para estos resultados se obtuvo que la fibra de plátano pretratada presenta propiedades hidrofóbicas ya que su ángulo de contacto es de $129.7^{\circ}$; mientras que la fibra de algodón no tuvo respuesta alguna frente a la caída de la gota agua y así dejó filtrar el agua, evitando medir su ángulo de contacto. Las fibras de plátano han demostrado tener una superficie auto-limpiante que aparte de generar una respuesta ante cualquier líquido también permite añadir características como resistencia a manchas químicas y a la generación de microorganismos en su estructura debido a que sus interacciones sólido-líquido son débiles, pero líquido-líquido tienden a ser muy fuertes (Felipe y Vicente, 2015).

Figura 6. Resultados de EDS de la Muestra 7. Inserto: fibra de plátano nanoestructurada con NPs de ZnO2 (Muestra 7) después del lavado

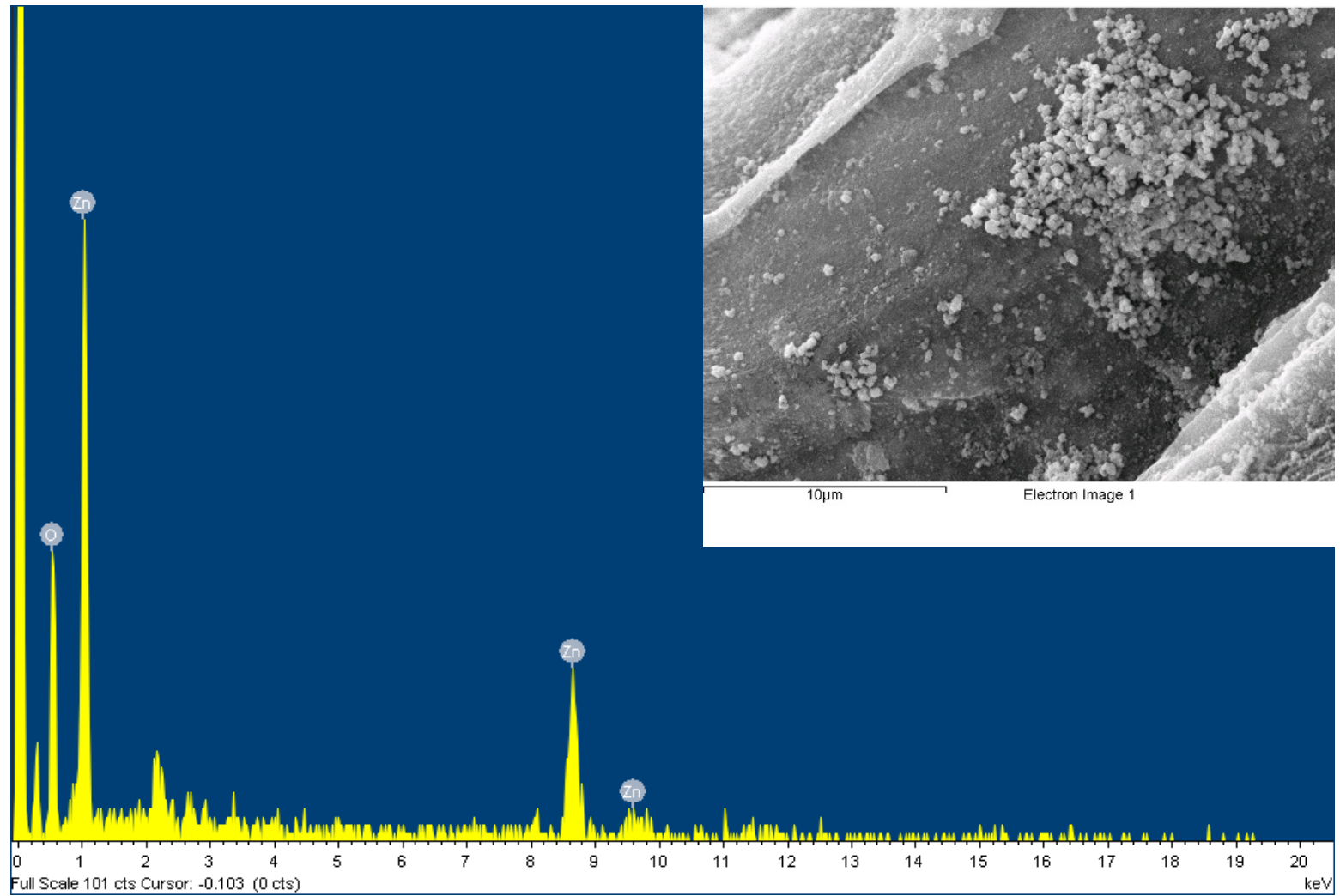


Figura 7. Recubrimiento de $\mathrm{NPs}_{\mathrm{ZnO}}$ después del lavado $\mathrm{M} 16$

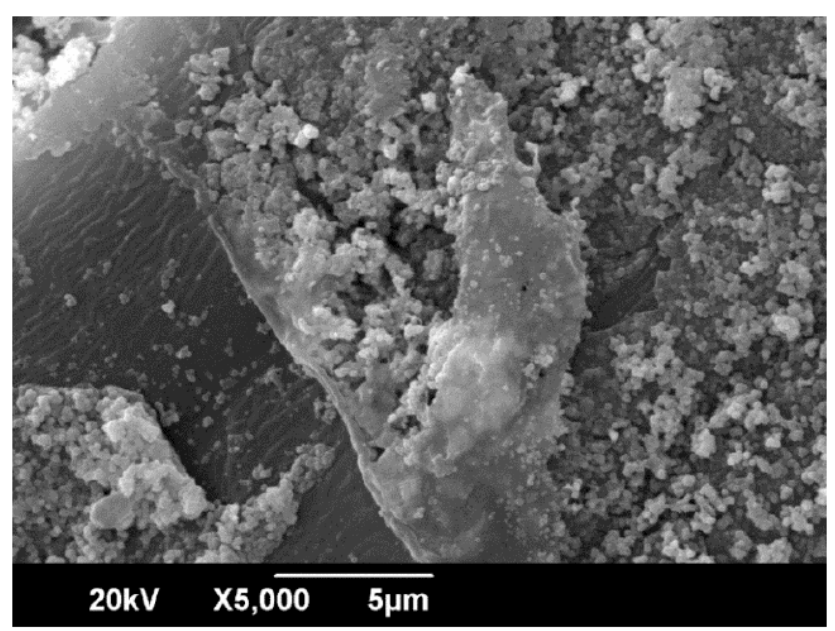

Luego de lavado con detergente, se observó (Figura 6) una disminución de NPs de $\mathrm{ZnO}_{2}$ sin embargo en su gran mayoría no tuvieron migración desde de la fibra. Mediante el software ImageJ (Schneider et al., 2012) se determinó la pérdida de NPs de ZnO2 como 47,58 $\%$ para Muestra 7, mediante la comparación entre la fibra nanoestructurada y luego de ser lavada.

Para la Figura 7 y Muestra 16, se observó que las nanopartículas tuvieron un desprendimiento después del lavado pero menor que el en la Figura 8. A pesar de la eficacia del detergente, se puede rescatar que una sección compacta de la fibra mantuvo las nanopartículas en su estructura. Para este caso la pérdida de NPs $\mathrm{ZnO}_{2}$ fue de un 18,34\%, mediante la técnica descrita anteriormente.

En cuanto a los resultados de las propiedades antibacteriales, la Figura 8 muestra que la fibra de algodón presentó menor inhibición a $E$. coli, mientras que la fibra de plátano (fibra pretratada) demuestra propiedades antibacteriales sin necesidad de recubrimiento, confirmando sus propiedades naturales antibacteriales. Dentro de las dos fibras de plátano nanoestructuradas tanto la Muestra 6 como la 7 presentan inhibición de los microorganismos, aunque ésta última presenta la mayor inhibición en comparación a todas las muestras estudiadas. La Tabla 5 resume los resultados medidos de halo de inhibición bacteriana $(\mathrm{mm})$ luego de que las fibras estuvieron en presencia de $E$. coli a una temperatura constante de $37^{\circ} \mathrm{C}$ durante 48 h. Para esta prueba se comprobó la capacidad antimicrobiana que presenta la fibra de plátano desde su punto inicial (fibra pretratada) y su capacidad ascendente cuando están nanoestructuradas. Por otro lado, se presenta la fibra de algodón que no presentó ningún tipo de frontera para su resistencia y con la nanoestructuración esta propiedad es robustecida.

Figura 8. Inhibición microbiana de la fibra de algodón empleada como testigo negativo (a), fibra pretratada (b), fibra nanoestructurada Muestra 7 (c), y fibra nanoestructurada Muestra 16 (d)

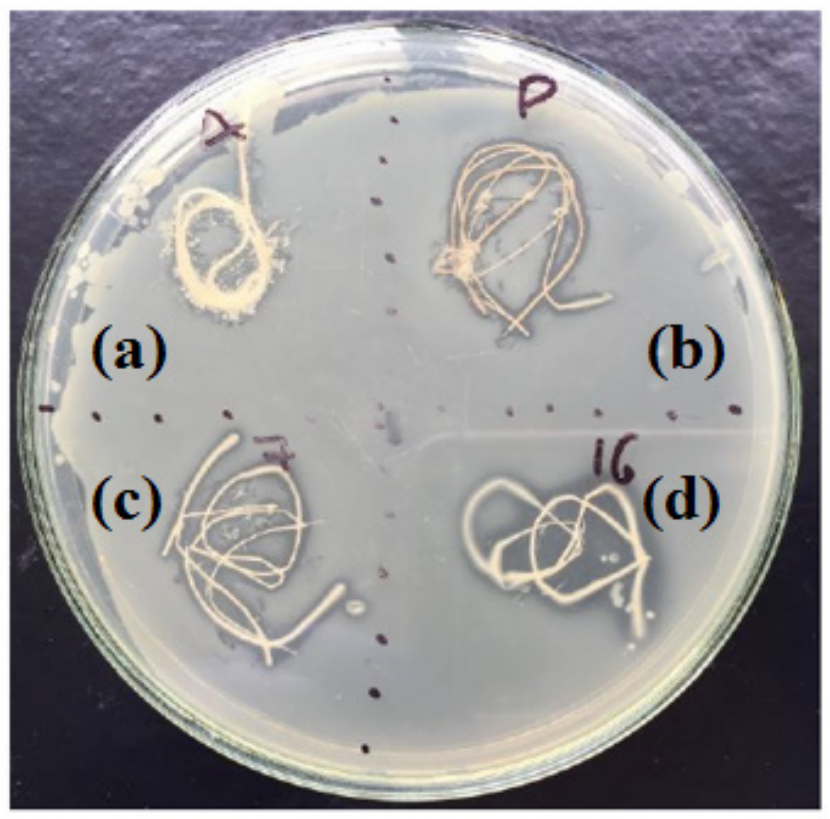

Tabla 5. Resultados obtenidos desde el centro de la fibra hasta el halo que produjo su actividad antimicrobiana

Fibra

Promedio de Longitud Halo Fibra-Respuesta (mm)

\begin{tabular}{lc}
\hline Algodón & 0 \\
Pretratada & $2,71+/-0,2$ \\
Muestra 7 & $3,85+/-0,2$ \\
Muestra 16 & $3,99+/-0,2$
\end{tabular}

Finalmente, mediciones de tensión (\%) y resistencia (cN/dTex) fueron determinadas para tres muestras: algodón, fibra de plátano sin y con nanoestructuración. La Figura 9 muestra el comportamiento a la tensión por cada una de las fibras escogidas. Es notorio que la 
fibra de plátano presenta buenas propiedades mecánicas en comparación con el algodón, lo que justificaría a la fibra de plátano como una buena opción en la industria textil. Por otro lado, la nanoestructuración permite mejorar la resistencia a la tensión que ya presenta la fibra de plátano por sí sola. La Tabla 6, que recoge los resultados numéricos de tensión (\%) y resistencia (cn/dtex), muestra que para ambas propiedades, la fibra de plátano nanoestructurada exhibe mejores resultados así: mejora la tensión del algodón y de la fibra de plátano en un $22 \%$ y $20,3 \%$, respectivamente; de igual manera, la presencia de las NPs de $\mathrm{ZnO}_{2}$ sobre la fibra de plátano mejora la resistencia del algodón y de la fibra de plátano en un $22 \%$ y $20,3 \%$, respectivamente en un $33,7 \%$ y $12 \%$, respectivamente.

Figura 9. Curva de tensión deformación (Stress-Strain) para algodón, fibra de plátano pretratada fibra de plátano nanoestructurada

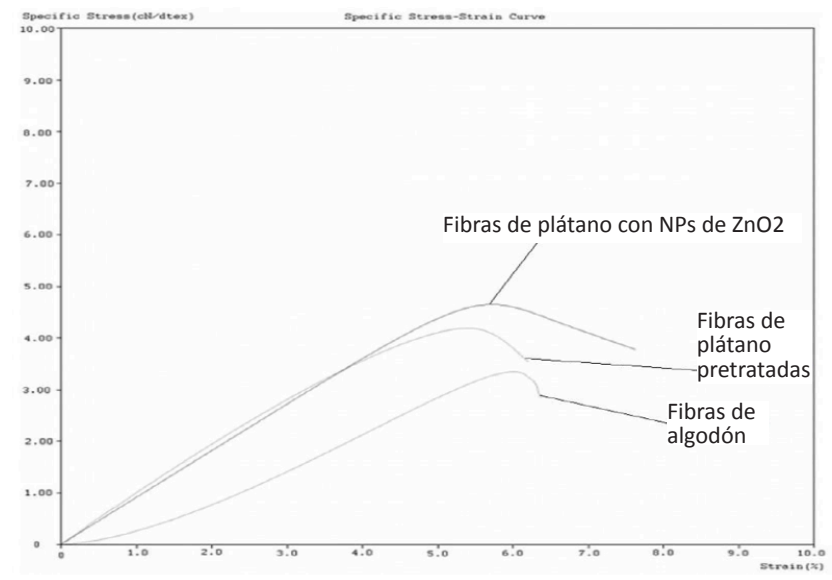

Tabla 6. Resultados de tensión para diferentes tipos de fibras: algodón (testigo), fibra pretratada y nanoestructurada con NPs de $\mathrm{ZnO}_{2}$

Fibra Tensión (\%) Resistencia (cn/dtex)

\begin{tabular}{ccc}
\hline Algodón & $6,138+/-0,3$ & $3,250+/-0,2$ \\
Pretratada & $6,302+/-0,3$ & $4,315+/-0,2$ \\
Nanoestructurada & $7,911+/-0,2$ & $4,901+/-0,4$
\end{tabular}

\section{CONCLUSIONES}

La investigación tuvo como objetivo nanoestructurar fibras provenientes de residuos del plátano con nanopartículas de peróxido de zinc ( $\mathrm{NPs}_{\text {de }} \mathrm{ZnO}_{2}$ ) como una propuesta de valor agregado en la industria textil. Sin embargo, también se tuvieron en cuenta factores de optimización en el proceso de la síntesis de las nanopartículas de hidróxido de zinc. El diseño experimental, empleado para conocer las variables de optimización en el proceso de la síntesis de las NPS, permitió conocer la concentración óptima de peróxido de hidrógeno, el tiempo posterior a la adición del acetato de zinc di-hidratado $\left(\left(\mathrm{CH}_{3} \mathrm{COO}\right) 2.2 \mathrm{H}_{2} \mathrm{O}\right)$ y el tiempo de sonicación que fueron $30 \%, 5,56$ min y $41,39 \mathrm{~min}$, respectivamente. Los resultados obtenidos por el diseño experimental garantizaron un tamaño de partícula entre $90 \mathrm{~nm}$ - $100 \mathrm{~nm}$. Se comprobó la capacidad hidrofóbica de la fibra de plátano nanoestructurada, con un ángulo de contacto promedio de $129.7^{\circ}$. Además, se destaca su capacidad antimicrobiana a $E$. coli y su resistencia a la tensión mejorada con respecto a la fibra de algodón. Por todo lo anterior, la fibra de plátano nanoestructurada es potencialmente una opción en la industria textil, que da valor agregado a residuos agroindustriales provenientes de los cultivos de plátano con actuales problemas de disposición final.

\section{AGRADECIMIENTOS}

Los autores agradecen a la Universidad de Bogotá Jorge Tadeo Lozano por el apoyo de los docentes y técnicos del Departamento de Ingeniería y de la Escuela de Diseño, así como su infraestructura.

\section{REFERENCIAS}

Alarcón, M., López, J., y Restrepo, D. (2013). Caracterización de la Funcionalidad Tecnológica de una Fuente Rica en Fibra Dietaria Obtenida a partir de Cáscara de Plátano. Revista Facultad Nacional de Agronomia - Medellín, 66(1), 6959- 
6968. Alonso, J. V. (2015). Manual control de calidad en productos textiles y afines. Madrid: Ediciones Escuela Técnicas Superior de Ingenieros Industriales.

Amestoy Alonso, J. (2009). El Planeta Tierra en peligro : calentamiento global, cambio climático, soluciones. Editorial Club Universitario.

Canché-Escamilla, G., De los Santos-Hernández, J. M., Andrade-Canto, S., y Gómez-Cruz, R. (2005). Obtención de Celulosa a Partir de los Desechos Agrícolas del Banano. Información Tecnológica, 16(1), 83-88, https://doi.org/10.4067/S071807642005000100012

Colonia, R., Martínez, V., y Solís, J. (2013). Síntesis de nanopartículas de $\mathrm{ZnO} 2$ empleando ultrasonido: caracterización estructural y morfológica para aplicaciones bactericidas. Revista de La Sociedad, (79)2, 126-135, disponible en http://www.scielo. org.pe/scielo.php?script=sci_arttext\&pid=S1810$634 \times 2013000200005$

Córdoba Padilla, M. (2011). Formulación y evaluación de proyectos. Ecoe Ediciones.

Departamento Administratico Nacional de Estadistica - DANE (2014). Boletín mensual SIPSA - Agosto 2014. Disponible en http://www.dane.gov.co/ index.php/52-espanol/noticias/noticias/3093boletin-mensual-sipsa-agosto-de-2014

Felipe, A., y Vicente, J. (2015). Manual Control de calidad en productos textiles y afines. Ediciones Escuela Técnicas Superior de Ingenieros Industriales, España.
Herrera, M. L. (1999). Pruebas de sensibilidad antimicrobiana Métodología de laboratorio. Revista Médica Del Hospital Nacional de Niños Dr. Carlos Sáenz Herrera, 34, 33-41.

León, G., González, M., Mercado, C., Eduardo, S., y Ternera, G. (2012). Metodología para determinar la factibilidad de proyectos inmobiliarios en el municipio de sabaneta para estratos 4 y 5 . Universidad de Medellín.

Ovalle, S. A., Blanco-Tirado, C., y Combariza, M. Y. (2013). Síntesis in situ de nanopartículas de plata sobre fibras de fique. Revista Colombiana de Química, 42(1), 1-27.

Quesada k, Alvarado P, y Sibaja R, V. J. (2005). Utilización de las fibras del rastrojo de piña (Ananas cosmusus, variedad champaka) como material de refuerzo en resinas de poliéster. Revista Iberoamericana de Polimeros, 6(2), 22.

Rajendra, R., Balakumar, C., Ahammed, H., Jayakumar, S., Vaideki, K., y Rajesh, E. (2010). Use of zinc oxide nano particles for production of antimicrobial textiles. International Journal of Engineering, Science and Technology, 2(1), https://doi.org/10.4314/ijest.v2i1.59113

Schneider, C. A., Rasband, W. S., y Eliceiri, K. W. (2012). NIH Image to ImageJ: 25 years of image analysis. Nature Methods, 9(7), 671-5. 